\title{
Laryngotracheal invasive thyroid cancer management
}

\begin{abstract}
Thyroid cancer incidences have drastically risen over the past few decades, probably due to early diagnostic facilities. However, Invasive thyroid cancers are rare the criticality of neighbouring structures sometimes may limit the extent of surgery. But, adjunct treatments with radio iodine therapy and in some instances, external beam radiotherapy tend to improve overall survival there is no match to, the scenario when proper disease clearance is attained surgically. Hence, this case report is being presented along with available review of literature.
\end{abstract}

Keywords: thyroid malignancy, larynx infiltration, laryngectomy, invasive cancers
Volume 7 Issue 3 - 2017

\section{Deepak Janardhan, Shaji Thomas, Virendra \\ Kumar Tiwari, Sunil kumar}

Department of Surgical Oncology, India

Correspondence: Deepak Janardhan MS ENT Fellowship in Head and Neck Surgery Senior Resident Department of Surgical Oncology Head and Neck Division Regional Cancer Centre Trivandrum Kerala, India, Tel 91-9961319859, Email dr.deepakjanardhan@gmail.com

Received: March 16, 2017| Published: June 05, 2017
Abbreviations: TSH, thyroid-stimulating hormone; RLN, recurrent laryngeal nerve; CECT, contrast enhanced computed tomography; MRI, magnetic resonance imaging; CT, computed tomography

\section{Introduction}

Invasive thyroid cancers, though not uncommon, have a reported incidence of around 6-13\%. Invasion into the neighbouring critical structures, either directly or extranodally, indicates the aggressive nature of the tumour. Adequate awareness regarding the same, aids in planning optimal diagnostic strategies for adequate surgical clearance. This case report is published in view of the rarity of laryngotracheal invasion of thyroid cancers ( 0.7 to $1.56 \%$ as per literature).

\section{Case report}

A 56year old male, with no co-morbidities, was diagnosed with papillary carcinoma thyroid. He underwent total thyroidectomy with modified radical neck dissection followed by radio iodine ablation in 2008. Since then, he was on regular follow up, was on thyroid medications and calcium supplementation for permanent hypocalcaemia. He had a disease free period of 8years. In 2015, his Thyroglobulin values had risen to 259. Further evaluation revealed a heterogeneous nodule, anterior to the larynx with central compartment nodes (Figure 1). In view of partial infiltration into the larynx, he was advised total laryngectomy with central compartmental nodal mass clearance in June 2016 with a small possibility of shaving off the tumour and preserving larynx. The patient deferred surgery for alternate treatment options and presented 8months later with progression of disease. On examination, a $4 \times 3 \mathrm{cms}$ hard swelling in the anterior neck, fixed to the thyroid cartilage was noted. Left level 3 node of $2 \times 1 \mathrm{cms}$ was palapable. Nasopharyngolaryngoscopy revealed left vocal cord palsy and no mucosal lesions in larynx or trachea. CT revealed enhancing solid mass anterior to larynx, infiltrating the thyroid cartilage, measuring $32 \times 24 \mathrm{~mm}$ (Figure 2) and irregular calcified nodal mass in the right upper paratracheal region of size $24 \times 18 \mathrm{~mm}$, along with few well defined rounded nodular opacities noted in both lungs of size 3-6 mm, possibly metastasis. Treatment options were discussed and he was taken up for Total laryngectomy with central compartment neck dissection with bilateral posterolateral selective neck dissection. Intra operatively, mucosal bulge was noted in the anterior subglottic wedge (Figure 3). His post op period was uneventful and was he initiated on nasogastric tube feeds on
POD-01 and normal oral feeds on POD-7. His final histopathology report confirmed papillary carcinoma of thyroid, infiltrating thyroid cartilage of larynx. He has been initiated on thyroid suppression and is scheduled for radio iodine scan and thyroglobin assessment with a possible need for Radio iodine ablation.

\section{Discussion}

The worldwide incidence of thyroid malignancies has shown rising trends, over the few past decades. This is perhaps due to increased patient awareness of and easy availability and accessibility of diagnostic facilities. The excellent prognosis associated with well differentiated thyroid cancers, is further rewarding for both the treating surgeon and the patient alike. However, age, extra thyroidal extension, histology and distant metastasis are prognosticative, ${ }^{1}$ dropping 10 year survival rates from 91 to $45 \% .^{2}$ The incidence of invasive thyroid cancers range from $6-13 \%{ }^{1-4}$ The common sites in the order of decreasing frequency are strap muscles (53\%), Recurrent laryngeal nerve (RLN) (47\%), trachea (37\%), carotid sheath structures $(30 \%)$, esophagus (21\%) and rarely larynx (12\%). This is either by the tumour per say or by extra nodal extension. ${ }^{5}$ Thyroid malignancies with laryngotracheoesophageal infiltration can present with hoarseness, stridor, hemoptysis or dysphagia. Fibreoptic laryngoscopy can reveal vocal cord palsy, which alerts the surgeon regarding the invasive nature of the disease. Direct pressure or infiltration of the RLN can be the cause. ${ }^{6}$ The ultrasonological identification of tracheal invasion has a sensitivity of $42-91 \%{ }^{7-9}$ Contrast enhanced CT continues to remain as the ideal investigation of choice to plan surgery, except for the contrast interfering with radio Iodine therapy. In such cases, MRI gives better information with regard to tracheal, esophageal and recurrent laryngeal invasion. Other cardinal diagnostic tools in the armamentarium include barium swallow followed by esophagoscopy and bronchoscopy. The importance of identifying invasiveness of thyroid malignancies lies in their correlation with aggressiveness of the tumour, poorer histology, doubtful radio iodine avidity and higher possibility of distant metastasis.

Shin et al., ${ }^{10}$ classified tracheal involvement in invasive thyroid cancers into 4 groups: Type-1: abutment, II-invades cartilage, IIIinvolves lamina propria and IV-when visualized intralaryngotracheally even as small as a mucosal bulge. ${ }^{10}$ Recurrence rates advance from $8 \%$ to $79 \%$, in type II - IV cancers, following just shaving off procedures, with a corresponding precipituous fall of survival rates from 8.7 to 1.5 years. ${ }^{11}$ Lack of expertise in conservation 
laryngeal surgeries or underestimation of disease aggressiveness, might perhaps result in such compromising treatment decisions perioperatively.

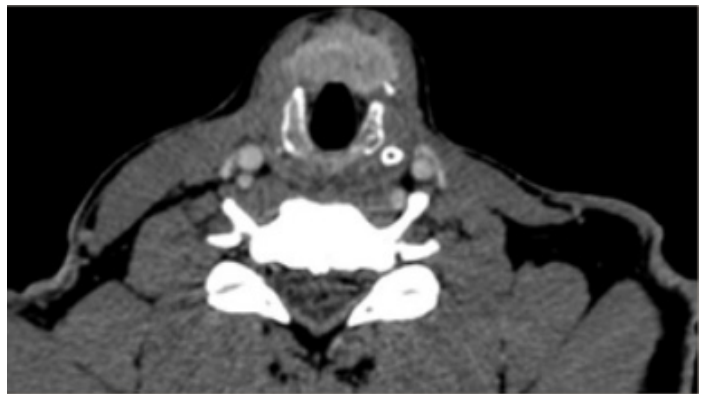

Figure I Heterogenously enhancing nodular lesion anterior to the cricothyroid membrane with partial laryngeal infiltration on a contrast enhanced computed tomography

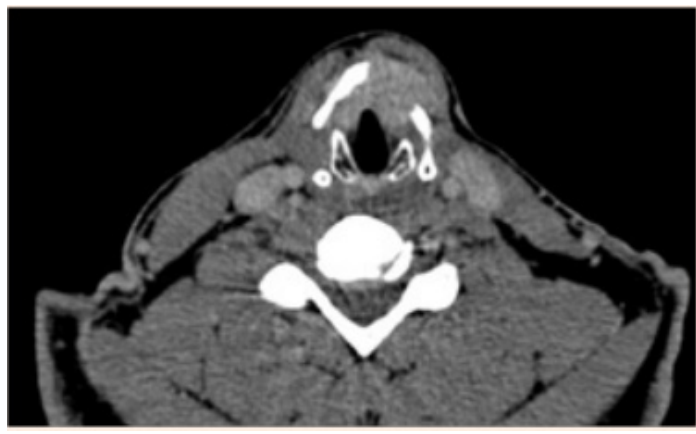

Figure 2 Contrast enhanced Computed tomography showing solid mass anterior to larynx infiltrating thyroid cartilage of size $32 \times 24 \mathrm{~mm}$.

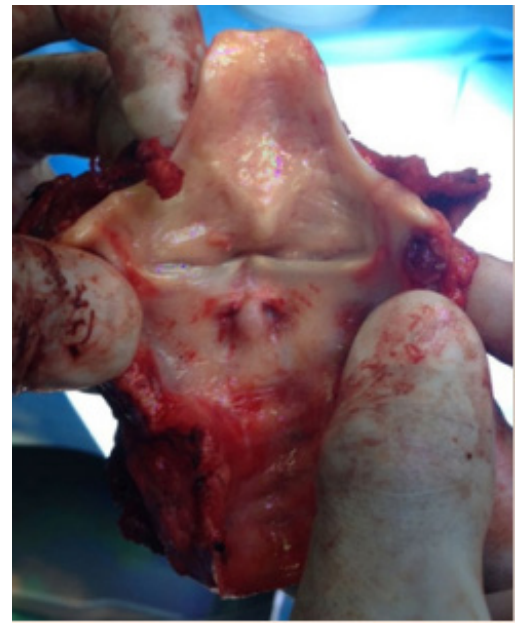

Figure 3 Laryngectomy specimen showing submucosal bulge in anterior subglottic wedge.

Surgical clearance of all gross disease in the neck remains the main stay of treatment. Critical structures like RLN, esophagus, trachea and carotid space structures often hinder aggressive approaches. Every attempt to preserve a functional RLN, by shaving off the tumour is advocated because, no survival difference has been reported after adjuvant RAI therapy in such patients when compared with nerve transaction. ${ }^{12-15}$ Esophageal infiltration worsens prognosis and though usually limited at muscular is layer, ${ }^{6,15}$ often warrants stent insertion for palliation. When laryngotracheal infiltration is noted, shaving the tumour off the critical structures is still widely practiced with the belief that radioiodine will melt the residual disease. EBRT offers some hope for non radio iodine avid tumours, although survival rates still remain poor. Proponents of aggressive approach with simultaneous partial or total laryngectomy and/or pharyngoesophageal resection and reconstruction with pedicled or free flaps, preach definitive survival difference. Conflicting reports on survival in such scenarios, are due to the varying definition of invasion, extent of resection, wide range of histology and different adjuvant therapies. Moreover, invasive nature with aggressive histology tends to leave behind micro residues. Hence, though morbid, the reported survival differences in literature warrants aggressive approach in appropriately selected patients for better DFS and OS. ${ }^{16-22}$ In the best interest of the patient, and the high chance of pulmonary metastasis, good local control is attained with comparatively lesser morbid total laryngectomy than partial procedures as in our case.

Adjuvant treatment with TSH suppression $(<0.1 \mathrm{mU} / \mathrm{L})$, in view of locoregional recurrence and metastasis, has improved local control with doubtful disease related survival advantage. Chemotherapy especially Doxorubicin has shown only $30-40 \%$ partial response with poor long term results. Molecular targetslike RET kinase inhibitors, $\mathrm{B}$ raf mutation targeting drugs and drugs restoring RAI uptake like retinoids and histone deacetylase etc are still in their infancy but may offer promising hope in the near future.

\section{Conclusion}

Invasive thyroid cancers, warrant aggressive treatment approach. But patient factors with distant metastasis burden should be taken into consideration prior to undertaking extensive and morbid resections followed by adjuvant treatment, for the best survival benefits. Laryngotracheal infiltration of thyroid cancers is definitely a rare entity. But when need arises, surgical team trained to simultaneously address the situation with either partial or total laryngectomy has to be in place. In our case, however, we opted for total laryngectomy in view of the progressive lung metastatic lesion which would further diminish the lung functions in the near future creating further morbidity.

\section{Acknowledgments}

None.

\section{Conflicts of interest}

Author declares there are no conflicts of interest.

\section{Funding}

None.

\section{References}

1. Shah JP, Loree TR, Dharker D, et al. Prognostic factors in differentiated carcinoma of the thyroid gland. Am J Surg. 1992;164(6):658-661.

2. Andersen PE, Kinsella J, Loree TR, et al. Differentiated carcinoma of the thyroid with extrathyroidal extension. Am J Surg. 1995;170(5):467-470.

3. Hay ID, McConahey WM, Goellner JR. Managing patients with papillary thyroid carcinoma: insights gained from the Mayo Clinic's experience of treating 2,512 consecutive patients during 1940 through 2000. Trans Am Clin Climatol Assoc . 2002;113:241-260.

4. Randolph GW, Kamani D. The importance of preoperative laryngoscopy in patients undergoing thyroidectomy: voice, vocal cord function, and the preoperative detection of invasive thyroid malignancy. Surgery. 2006;139(3):357-362. 
5. McCaffrey TV, Bergstralh EJ, Hay ID. Locally invasive papillary thyroid carcinoma: 1940-1990. Head Neck. 1994;16(2):165-172.

6. Machens A, Hinze R, Lautenschläger C, et al. Thyroid carcinoma invading the cervicovisceral axis: routes of invasion and clinical implications. Surgery 129(1): 23-28.

7. Shimamoto K, Satake H, Sawaki A, Ishigaki T, Funahashi H, et al. (1998) Preoperative staging of thyroid papillary carcinoma with ultrasonography. Eur J Radiol . 2001;29(1):4-10.

8. Chisato Tomoda, Takashi Uruno, Yuuki Takamura, et al. Ultrasonography as a method of screening for tracheal invasion by papillary thyroid cancer. Surg Today. 2005;35(10):819-822.

9. Yamamura N, Fukushima S, Nakao K, et al. Relation between ultrasonographic and histologic findings of tracheal invasion by differentiated thyroid cancer. World J Surg. 2002;26(8):1071-1073.

10. Shin DH, Mark EJ, Suen HC, et al. Pathologic staging of papillary carcinoma of the thyroid with airway invasion based on the anatomic manner of extension to the trachea: a clinicopathologic study based on 22 patients who underwent thyroidectomy and airway resection. Hum Pathol. 1993;24(8):866-870.

11. Nishida T, Nakao K, Hamaji M. Differentiated thyroid carcinoma with airway invasion: indication for tracheal resection based on the extent of cancer invasion. J Thorac Cardiovasc Surg . 1997;114(1):84-92.

12. Nishida T, Nakao K, Hamaji $M$, et al. Preservation of recurrent laryngeal nerve invaded by differentiated thyroid cancer. Ann Surg. 1997;226(1):85-91.

13. Tanaka K, Sonoo H, Yamamoto Y, et al. Analyses of the outcome of locally invasive papillary thyroid carcinomas. Thyroid. 1999;9(10):1017-1022.
14. Chiang FY, Wang LF, Huang YF, et al. Recurrent laryngeal nerve palsy after thyroidectomy with routine identification of the recurrent laryngeal nerve. Surgery. 2005;137(3):342-347.

15. Falk SA, McCaffrey TV. Management of the recurrent laryngeal nerve in suspected and proven thyroid cancer. Otolaryngol Head Neck Surg. 1995;113(1):42-48.

16. Friedman M. Surgical management of thyroid carcinoma with laryngotracheal invasion. Otolaryngol Clin North Am. 1990;23(3):495-507.

17. Fujimoto Y, Obara T, Ito Y, et al. Aggressive surgical approach for locally invasive papillary carcinoma of the thyroid in patients over fortyfive years of age. Surgery. 1986;100(6):1098-1107.

18. Grillo HC, Suen HC, Mathisen DJ, et al. Resectional management of thyroid carcinoma invading the airway. Ann Thorac Surg. 1992;54(1):3-9.

19. Grillo HC, Zannini P. Resectional management of airway invasion by thyroid carcinoma. Ann Thorac Surg . 1986;42(3):287-298.

20. Kim KH, Sung MW, Chang KH, et al. Therapeutic dilemmas in the management of thyroid cancer with laryngotracheal involvement. Otolaryngol Head Neck Surg. 2000;122(5):763-767.

21. Friedman M, Danielzadeh JA, Caldarelli DD. Treatment of patients with carcinoma of the thyroid invading the airway. Arch Otolaryngol Head Neck Surg. 1994;120(12):1377-1381.

22. Gaissert HA, Honings J, Grillo HC, et al. Segmental laryngotracheal and tracheal resection for invasive thyroid carcinoma. Ann Thorac Surg. 2007;83(6):1952-1959. 